\title{
Factors Controlling Oxophilicity and Carbophilicity of Transition Metals and Main Group Metals
}

\author{
Gbolade O. Kayode, ${ }^{\text {a }}$ Matthew M. Montemore. *a \\ ${ }^{a}$ Department of Chemical and Biomolecular Engineering, Tulane University, New Orleans, LA \\ 70118, USA
}

\begin{abstract}
The strength of interaction between a metal and oxygen and/or carbon is a crucial factor for catalytic performance, materials stability, and other important applications. While these are fundamental properties in materials science, there is no general understanding of what makes a metal oxophilic or carbophilic, especially for main group metals. In this work, we elucidate the factors that control how oxophilic or carbophilic a metal is by creating a predictive model and applying it to a variety of data sets for transition metals and main group metals, including DFTcalculated adsorption energies and experimental formation energies. Our model is easily interpretable and accurately describes oxophilic and carbophilic trends across different regions of the periodic table. This model captures the ionic contribution to bonding, the adsorbate-sp contribution to bonding, and the adsorbate-d contribution to bonding by using the reduction potential, matrix coupling elements, band centers, and band filling. For transition metals, the adsorbate-surface $d$ coupling is the major factor that determines oxophilicity relative to carbophilicity. For metals that do not contain $d$ electrons either in their core or valence shell $(\mathrm{Li}$, $\mathrm{Be}, \mathrm{Na}, \mathrm{Mg}, \mathrm{Al}, \mathrm{K}$, and $\mathrm{Ca}$ ), the reduction potential and the adsorbate-surface $s$ coupling are the major factors. As a simple application, we demonstrate the utility of oxophilicity and carbophilicity in rapidly screening metal dopants for improved selectivity for ethylene epoxidation on silverbased catalysts. Using our model, we established a direct relationship between the electronic
\end{abstract}


properties of the metal dopants and their selectivity for ethylene epoxidation. The results suggest that transition metals with high adsorbate-surface $d$ coupling and main group metals with low adsorbate-surface $s$ coupling are good silver-dopant candidates for this reaction. Overall, the improved linkage between a metal's electronic structure and its interaction with carbon or oxygen will be broadly useful in design of functional materials for a variety of applications.

\section{Introduction}

Improved understanding of the interaction between metals and carbon or oxygen is crucial in materials science, for both fundamental and technological reasons. ${ }^{1-9}$ For example, these interactions are important for catalytic processes, such as methane steam reforming ${ }^{8}$, acetylene hydrogenation, ${ }^{1}$ ethylene epoxidation, ${ }^{2-4}$ hydrogenation of unsaturated aldehydes, ${ }^{6,7}$ and $\mathrm{CO}_{2}$ conversion reactions ${ }^{5}$. Hence, the strength of interaction between metal catalysts and oxygenates or carbonaceous species is widely used to screen catalysts or rationalize catalytic performance.

In addition to affecting catalytic activity and selectivity, oxophilicity and carbophilicity have wide-ranging implications on materials stability. For example, these factors can explain why gold pieces of jewelry do not tarnish ${ }^{10}$ and why certain metals coke more easily than others ${ }^{11}$. More generally, an improved understanding of these properties could aid in designing surfaces that are stable under aqueous or oxidizing conditions ${ }^{12,13}$. Further, in minerology, ${ }^{14-16}$ oxophilic and carbophilic properties play a major role in the formation of fine oxides, carbonate minerals and carbide minerals. Therefore, understanding the inherent affinity of metals towards oxygen and carbon is of fundamental and broad-ranging importance.

Although oxophilicity and carbophilicity have been studied within certain classes of metals, ${ }^{17-21}$ their overall trend across metals from different regions of the periodic table has not been elucidated. In particular, trends in main group metals have received very little attention, 
despite their importance in many technologies. Understanding these trends and the factors that control them would enable and improve the rational design of materials for a variety of applications. Further, having a consistent database of oxophilicity and carbophilicity values, as we provide here, allows fast, approximate screening of elements for a particular application without requiring further calculations.

Many studies have explored factors that influence the oxophilicity and carbophilicity of metals, with the most attention given to transition metals. The widely known $d$-band model ${ }^{22-24}$ suggests that the metal's $d$-band center and the matrix coupling element between surface and adsorbate electronic states are the prime factors responsible for the adsorption strength of various species on metals. This has been corroborated by recent studies ${ }^{20,25,26}$, as the $d$-band center and the coupling matrix element have proven to be important descriptors when considering the adsorption of hydrocarbons and oxygenates. These properties are related to hybridization-which affects covalent bonding — and Pauli repulsion. In addition to these properties, some previous studies have proposed that properties related to charge transfer, such as the electronegativity ${ }^{17}$ or reduction potential, ${ }^{21}$ can affect adsorption energies. Electronegativity has been found to be particularly useful in explaining trends of bond dissociation energies between metal atoms and O or S. ${ }^{18}$ However, these previous studies have not examined trends across both transition metals and maingroup metals, such that a broader understanding has not been achieved.

Studies on bulk formation energies, ${ }^{27-29}$ another useful quantifier of oxophilicity and carbophilicity, have shown that the formation energies of binary metal compounds (oxides, carbides, nitrides, and sulfides) can be dependent on the metal's properties. For instance, properties related to charge transfer such as electronegativity, ionization energies, electron affinity, and atomic electric polarizabilities have been shown to be related to the stability of some of these 
binary metallic compounds. ${ }^{27}$ Another study has shown that the reactivity of surface oxygen atoms in oxides is strongly correlated to the average $2 p$-state energy $\left(\bar{\varepsilon}_{2 p}\right) \cdot{ }^{30}$ Also, many machine learning (ML) approaches have also been used to predict formation energies of these binary metallic compounds with good accuracy ${ }^{31-33}$. However, most of these models are focused on accuracy and are not interpretable enough to draw out conclusions about the underlying physics.

Because previous studies focused on metals within certain regions of the periodic table (mostly transition metals), a more general understanding remains elusive. For instance, the correlation between oxophilicity and electronegativity only holds for transition metals (see Figure S1). Also, some of these studies only consider bulk metals. For catalysis, it is important to extend the scope to include metal surfaces. From our previous work on transition metals, ${ }^{19,20,34,35}$ we discovered that adsorption energies of many species on transition metals, including hydrocarbons and oxygenates, can be predicted from just a few electronic properties. These properties include the d-band center, d-band filling, number of $p$ electrons, and the matrix coupling element.

For this present work, we extend our scope of study to include metals from other regions of the periodic table. We quantify oxophilicity and carbophilicity using adsorption energies and experimental formation energies to capture surface and bulk perspectives respectively. We developed a general and interpretable model that accurately predicts and captures oxophilic and carbophilic trends within different regions of the periodic table, including transition metals and non-transition metals. We found the reduction potential to be a better representation of ionic contributions than electronegativity, especially when considering metals from different regions of the periodic table. As an application, we applied our model to understand selectivity trends and screen for dopants for ethylene epoxidation on silver-based catalysts. 


\section{Methods}

All experimental formation energies used in this work were extracted from the Open Quantum Materials Database (OQMD) ${ }^{36}$ The reduction potentials $R_{P}$, defined as the intrinsic tendency of a metal to lose or gain an electron, were compiled from Quantitative Chemical Analysis. ${ }^{37}$ This quantity is measured under standard conditions in an electrochemical cell containing a standard hydrogen electrode (SHE) and the metal to be investigated. The coupling element to the $d$ and $s$ band were computed as follows:

$$
\begin{aligned}
V_{a d}^{2} & =\sum_{j} \frac{\left(r_{d}^{i} r_{d}^{j}\right)^{\frac{3}{2}}}{d_{i j}^{7}} \\
V_{a s}^{2} & =\sum_{j} \frac{1}{d_{i j}^{4}}
\end{aligned}
$$

These equations and the $d$-orbital radii $\left(r_{d}\right)$ used in Equation 1 were taken from previous work, ${ }^{38}$ with values for metals in groups $12-15$ calculated using linear extrapolation. The atom-atom distances $\left(d_{i j}\right)$ were computed using the carbon-metal distance for $\mathrm{CH}_{3}$ on the top site, as shown in our previous work ${ }^{19,20,35}$. The values of all quantities used in our final model, including matrix coupling elements and the reduction potentials, are given in the Supporting Information.

Calculations. We performed density functional theory (DFT) calculations using the Vienna ab initio Simulation Package (VASP). ${ }^{39,40}$ These calculations involved the use of the PW91 exchangecorrelation functional ${ }^{41}$ and the projector augmented-wave method. ${ }^{40,42}$ An energy cut-off of 396 $\mathrm{eV}$ and a 7 x 7 x 1 Monkhorst-Pack ${ }^{43}$ k-point grid were also used. Each adsorbate $\left(\mathrm{O}, \mathrm{C}, \mathrm{OH}, \mathrm{CH}_{3}\right)$ was positioned in a $3 \times 3$ cell with four layers of metal with the lowest two layers fixed at their bulk positions. We utilized spin polarization for magnetic metals ( $\mathrm{V}, \mathrm{Cr}, \mathrm{Mn}, \mathrm{Fe}, \mathrm{Co}, \mathrm{Ni})$. In addition, density of states calculations were carried out by performing a single-point calculation 
with tetrahedral smearing using a smearing width of $0.01 \mathrm{eV}$. For this calculation, the charge density was fixed, and the k-point mesh was increased to 19 × 19 x 1 with 324 total bands. The DOS was sampled over intervals on the order of $10^{-3} \mathrm{eV}$ and the band centers were calculated by projecting the wave function on atom-centered spherical harmonics within a cut-off radius and taking the first moment of the entire band for a given atom. For metals containing no $d$ electrons in core or valence, the d-band center was set to 0 ; for all others the calculated value was used. For metals with a very low d-band center $(\mathrm{Zn}, \mathrm{Cd}$ and $\mathrm{Hg})$, the d-band center was set to $-4 \mathrm{eV}$, as states that are very low in energy should have only a small effect on chemical bonding. The DOS was calculated for materials in both the fcc structure and the ground-state structure, and there was little difference in the electronic structure properties; therefore, we used electronic structure properties from fcc(111) surfaces in all cases.

\section{Results \& Discussion}

\subsection{Data.}

For solid-state metals, there are two broad ways to measure oxophilicity and carbophilicity. When considering surface oxidation, catalysis, and interface reactivity, oxophilicity and carbophilicity are often measured by the strength of the interaction between the material's surface and oxygen-containing or carbon-containing species, i.e., adsorption energies. When considering bulk oxidation, synthesis of bulk materials, and materials stability in harsh environments, it is more useful to consider oxophilicity and carbophilicity as the tendency of materials to form oxides or carbides. This can be quantified by formation energies. In this work, we examined both perspectives by using $\mathrm{O}, \mathrm{OH}, \mathrm{C}$, and $\mathrm{CH}_{3}$ adsorption energies as well as oxide and carbide formation energies. We calculated $\mathrm{O}, \mathrm{OH}, \mathrm{C}$, and $\mathrm{CH}_{3}$ adsorption energies on all pure metals 
except lanthanides and actinides (46 total metals). For $\mathrm{OH}$ and $\mathrm{CH}_{3}$, we used the fcc(111) surface configuration for the metals, with $\mathrm{OH}$ and $\mathrm{CH}_{3}$ adsorbed on the top site. This gives an intrinsic measure of C-M and O-M bond strengths without any variation due to changes in adsorption site or surface geometry. Hence, these quantities may be particularly useful when understanding intrinsic properties of metals or when considering alloying a metal into a given structure. Meanwhile, for $\mathrm{O}$ and $\mathrm{C}$, we used the metals' ground-state crystal structures, with $\mathrm{O}$ and $\mathrm{C}$ adsorbed on the lowest energy site. This includes changes in adsorption site and crystal structure and may be more useful for considering the reactivity of the pure metal surfaces towards atoms.

The $\mathrm{OH}$ and $\mathrm{CH}_{3}$ adsorption energies show a few similarities in their trends across the periodic table (Figure 1). For example, adsorption is generally strongest towards the middle-left of the periodic table. Also, adsorption generally decreases down the group for alkali metals while it increases down the group for alkaline-earth metals. However, there are also clear differences in behavior between $\mathrm{OH}$ and $\mathrm{CH}_{3}$. For example, $\mathrm{OH}$ adsorbs most weakly to $\mathrm{Au}$ while $\mathrm{CH}_{3}$ adsorbs most weakly to $\mathrm{Ag}$. Further, $\mathrm{CH}_{3}$ adsorption strengthens when moving down the platinum-group metals Ni, Pd, Pt, while $\mathrm{OH}$ adsorption weakens. These trends are very similar to that of $\mathrm{O}$ and $\mathrm{C}$ across the periodic table (Figure S2). This comes as no surprise as $\mathrm{OH}$ and $\mathrm{CH}_{3}$ correlate strongly with $\mathrm{O}$ and $\mathrm{C}$ respectively (Figure S9). ${ }^{44} \mathrm{~A}$ notable difference, however, is that adsorption is weakest on $\mathrm{Hg}$ for $\mathrm{O}$ and $\mathrm{C}$, but weakest on $\mathrm{Au}$ and $\mathrm{Ag}$ for $\mathrm{OH}$ and $\mathrm{CH}_{3}$, respectively.

The quantitative difference between the $\mathrm{OH}$ and $\mathrm{CH}_{3}$ adsorption energies represents a simple measure of the oxophilicity relative to carbophilicity (Figure 1c). This difference can be important in catalytic performance. ${ }^{19}$ For example, Pt is very carbophilic relative to its oxophilicity, which can explain its susceptibility to CO poisoning. ${ }^{45}$ Metals towards the left of the periodic table are highly oxophilic relative to their carbophilicity, and to the right less oxophilic 
than carbophilic. Metals further down in a group are typically less oxophilic relative to their carbophilicity, with a few exceptions.

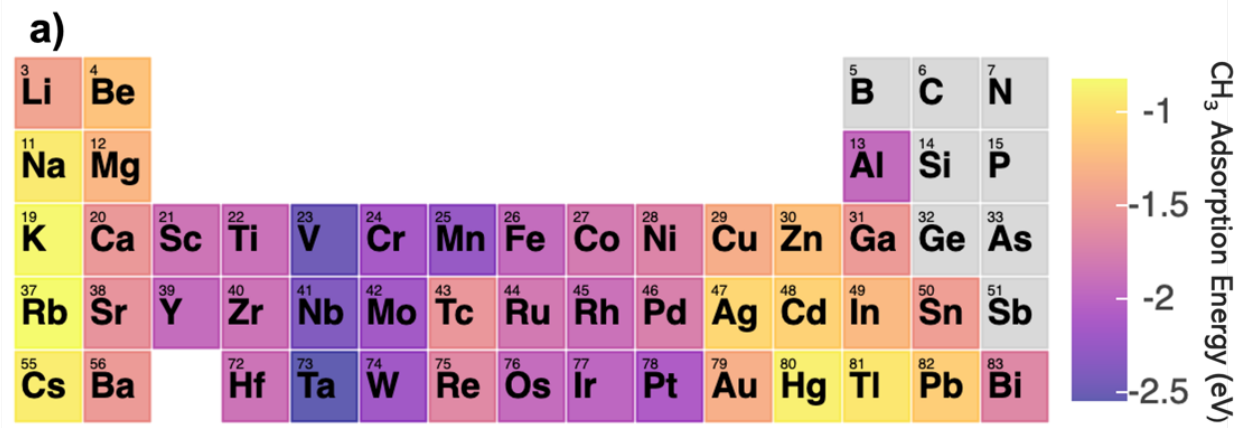

\section{b)}
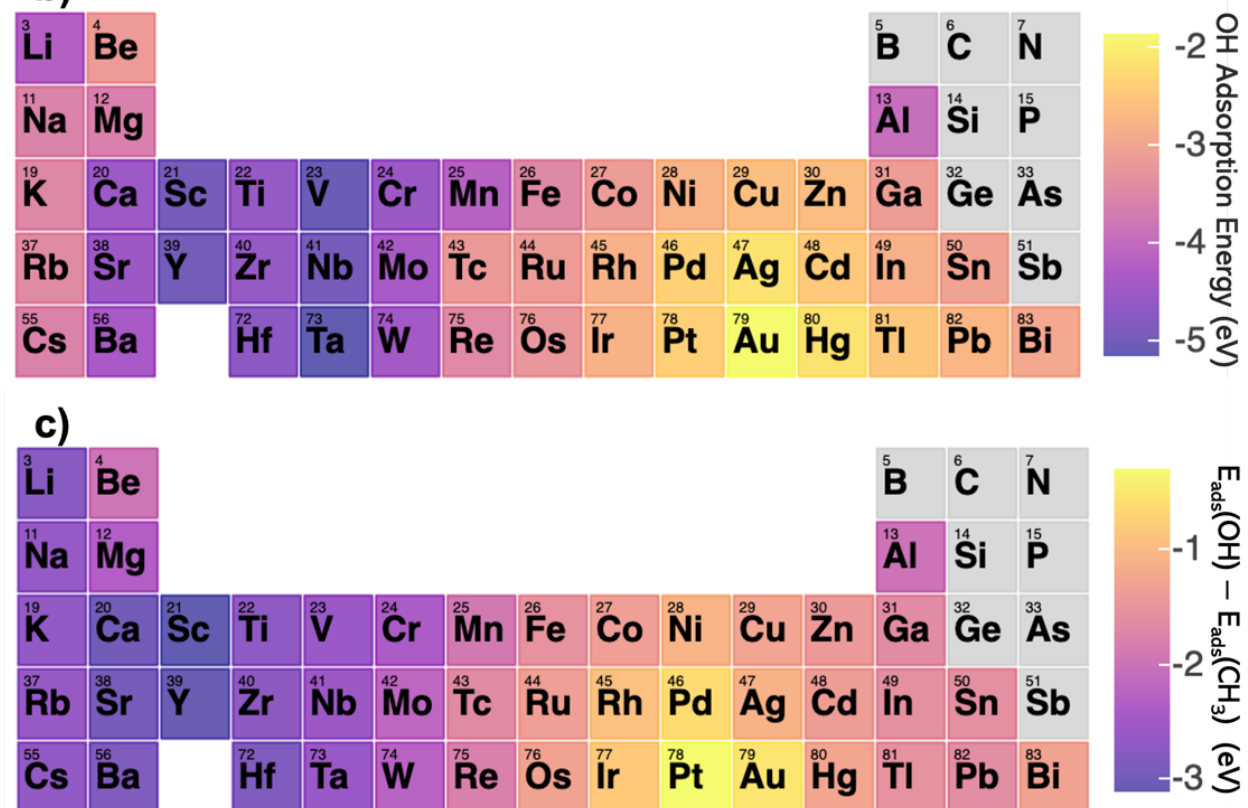

Figure 1 Periodic tables of the DFT-calculated, top-site $\mathrm{CH}_{3}$ and $\mathrm{OH}$ adsorption energies and their quantitative difference for all metals considered in this work.

To compare the two perspectives of oxophilicity and carbophilicity stated above, we examined the relationship between the adsorption energies and formation energies of various transition metal compounds (see Figure 2). We may expect a strong correlation between adsorption energies and formation energies of the same species. This correlation indeed holds in some cases; for example, the oxygen adsorption energy and oxide formation energy are strongly correlated $\left(\mathrm{R}^{2}\right.$ $=0.80 \pm 0.09)$. However, in other cases, like that of the carbon adsorption energy and the carbide 
formation energy, the correlation is weak $\left(R^{2}=0.47 \pm 0.36\right)$. Therefore, these two definitions can indeed be quite distinct in some cases.

The oxygen adsorption energy has the strongest correlation with all the formation energies, especially with the oxide and carbide formation energies (see Figure 2). Because of the strong correlation the oxygen adsorption energy has with the oxide formation energy $\left(\mathrm{R}^{2}=0.80 \pm 0.09\right)$ and carbide formation energy $\left(\mathrm{R}^{2}=0.83 \pm 0.25\right)$, we can infer that the surface properties that control the adsorption energy of oxygen on metal surfaces would likely also control the formation energy of oxides and carbides of the corresponding bulk metals. This suggests it may be possible to predict both formation energies and adsorption energies using a single set of properties.

In addition to the relatively strong correlation between the $\mathrm{O}$ adsorption energies and all formation energies, formation energies and adsorption energies are more likely to correlate if they involve the same or similar species. For example, methyl and carbon adsorption energies have almost no correlation with the sulfide, nitride, or oxide formation energies, but have some correlation with the carbide formation energy. Similarly, the hydroxyl adsorption energy correlates more strongly with the oxide formation energy than the other formation energies. Broadly, knowledge of formation energies can give some insight into adsorption energies (and vice versa), if care is taken to make the proper comparison.

We also examined the correlations among adsorption energies and among formation energies (Figure S9). Adsorption energies involving the same species, like hydroxyl and oxygen or methyl and carbon, correlate strongly with one another. However, those involving different species have weak correlations. For example, hydroxyl and carbon; oxygen and methyl; and hydroxyl and methyl all have weak correlations. These trends are expected based on previous work on transition metals. ${ }^{20,46}$ Among formation energies, we observe the strongest correlation between 
oxides and nitrides. Sulfides also correlate well with oxide and carbides, while the sulfide-nitride and carbide-oxide correlations are weak.

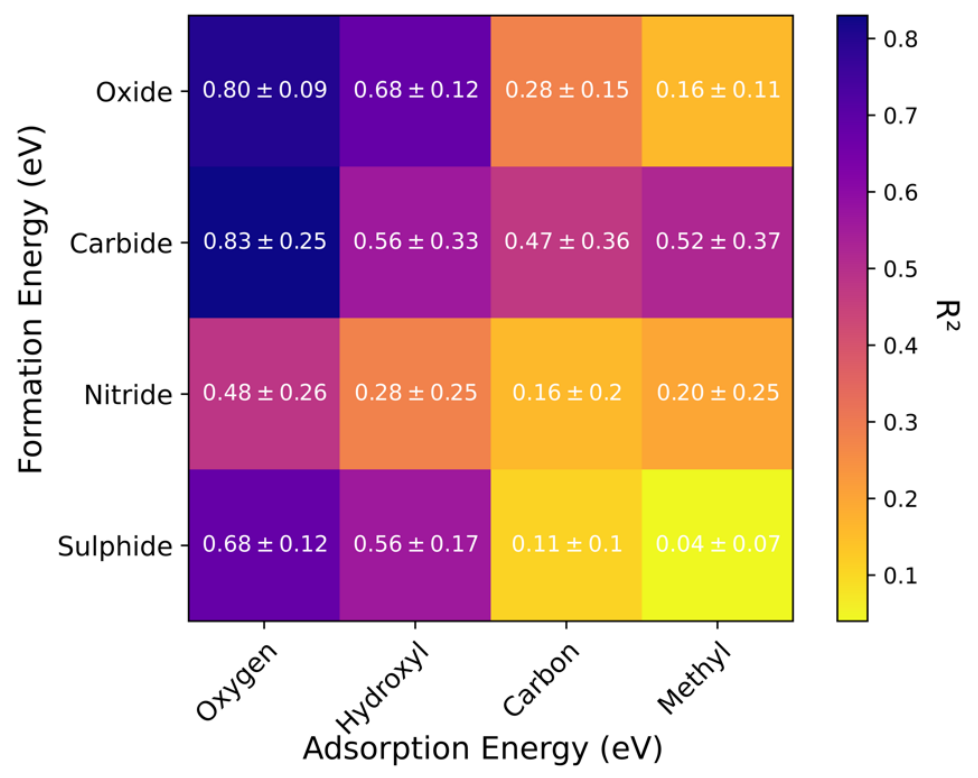

Figure 2 Correlations between calculated adsorption energies on metal surfaces and experimental formation energy of bulk metal compounds for various chemical species. The oxygen adsorption energy has the strongest correlation with all of the formation energies shown.

\subsection{Model development.}

To understand what properties control oxophilicity and carbophilicity, we developed a general and interpretable predictive model. Because our focus is on generality and interpretability, we chose a high-bias and low variance model, the linear model. Linear models are less prone to overfitting and have been used to produce interpretable models with relatively small data sets, as in our case here. In creating our model, we used data-driven techniques to identify more accurate models, and physical intuition to choose between models with similar accuracy. This has been successful in creating general models in previous work, ${ }^{19,20,35}$ and is particularly useful when considering multiple adsorbates that can have different adsorption energy ranges and hence different error magnitudes. This combined approach helps to mitigate the researcher bias that physically generated models alone can bring and also ensures that data-driven models have 
physical justifications. The result is a model that is accurate from a data perspective and highly interpretable from a physical perspective.

For the feature selection, we examined a total of 40 features (Table S1) for their predictive abilities. We first used a brute-force, best-subset approach to select potential feature sets for our final model. The brute-force approach for each adsorbate $\left(\mathrm{O}, \mathrm{C}, \mathrm{OH}, \mathrm{CH}_{3}\right)$ simply involved fitting linear models using every possible feature combination up to 6 features within our feature space and ranking them from most to least accurate. The most accurate model was somewhat different for each adsorbate. To solve this challenge, we considered the physical motivation for each potential set of features as well as their predictive capability across the various adsorbates. Hence, we selected the most important feature set for our final model from a physical perspective, while preserving accuracy. The final feature set included simple combinations of the d-band center $\varepsilon_{d}$, the adsorbate-surface $s$ coupling $V_{a s}^{2}$, the idealized band filling $f$, the adsorbate-surface $d$ coupling $V_{a d}^{2}$, and the metal's reduction potential $R_{p}$. The model inputs and their physical justification are shown in Table S2. Many of the properties in this equation are well-known from previous work in solid-state physics and adsorption, ${ }^{23,24,47}$ which again suggests that the model captures important physics. Therefore, a good compromise between accuracy, complexity, generality, and interpretability for oxophilicity and carbophilicity is given by

$$
E_{a d s / f o r m}=\alpha_{1}+\alpha_{2} \varepsilon_{d}+\alpha_{3} V_{a s}^{2}+\alpha_{4} V_{a s}^{2} f+\alpha_{5} V_{a d}^{2}+\alpha_{6} V_{a d}^{2} f+\alpha_{7} R_{p} f
$$

Qualitatively, we can divide the formation of a chemical bond into contributions from hybridization, Pauli repulsion, and charge transfer. The $R_{p} f$ term accounts for charge transfer (i.e., the ionic contribution to bonding), while the other terms capture the covalent contributions to bonding. Increasing the coupling between the adsorbate's electronic states and the metal's states 
increases the amount of attraction by hybridization, as well as the amount of Pauli repulsion. Repulsion is also expected to increase as bands become more full, as predicted by molecular orbital theory. The balance between these factors manifests in the fitting parameters, as discussed below. While most previous work on transition metals focuses heavily on the d-band, we found in previous work that the s and p electrons can affect adsorption on transition metals. ${ }^{19,48}$ Indeed, the d-band model envisions adsorbate states first coupling to surface $\mathrm{s}$ and $\mathrm{p}$ states, followed by interaction with the $\mathrm{d}$ states. When considering main-group metals in addition to transition metals, it is expected that the sp band will contribute even more significantly to the variations in adsorption energies, as shown in the model terms that include $V_{\text {as }}^{2}$.

We applied this model to the adsorption energies of $\mathrm{O}, \mathrm{OH}, \mathrm{C}$, and $\mathrm{CH}_{3}$ (Figure 3). The model parameters for the fits can be found in Table 1. The mean absolute error (MAE) and root mean squared percent error (RMSPE) are: for $\mathrm{O}, 0.43 \mathrm{eV}$ and $8.7 \%$; for $\mathrm{OH}, 0.23 \mathrm{eV}$ and $8.5 \%$, for $\mathrm{C}, 0.37 \mathrm{eV}$ and $8.2 \%$; and for $\mathrm{CH}_{3}: 0.17 \mathrm{eV}$ and $14.9 \%$. Adsorbates that bind more strongly to the surface-like $\mathrm{C}$ and $\mathrm{O}$ - have adsorption energies with larger magnitudes and larger variation, and hence they have a higher MAE. However, the RMSPE is much more constant, suggesting the model is roughly equally accurate over the various adsorbates. The overall MAE and RMSPE were $0.30 \mathrm{eV}$ and $10.6 \%$. We compared this current model with our previously developed model for transition metals ${ }^{20}$ and found our current model to be an improvement. For all species only on transition metals, the MAE/RMSPE are $0.16 \mathrm{eV}$ and $7.34 \%$ for our current model and $0.22 \mathrm{eV}$ and $10.05 \%$ for the previous model. We attribute this improvement largely to the inclusion of the ionic term $R_{p} f$. Our model is also comparable in accuracy with other ML models for transition metals even though we consider a wider array of metals. ${ }^{49}$ 


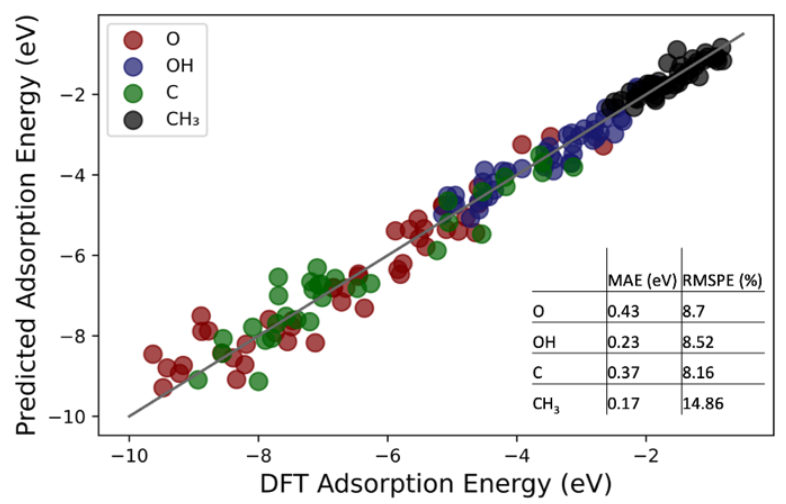

Figure 3 Parity plot between DFT-calculated adsorption energies and model-predicted adsorption energies for $\mathrm{O}, \mathrm{OH}, \mathrm{C}, \mathrm{CH}_{3}$ using Equation 3. MAEs and RMSPEs are given for each species.

To ensure our model is not overfitting, we varied the model complexity from one to six terms and performed 25\%-75\% test-train splits. For each number of terms, we chose the subset of terms from Equation 3 that gave the least error, performed one hundred test-train splits, and then averaged the results (Figure 4). We used the RMSPE because it is less biased towards imbalance in dataset ranges. The results show that the full six-term model (Equation 3) is not overfitting, as the test and train errors are similar, and the test error is still lower than that of a five-term model.

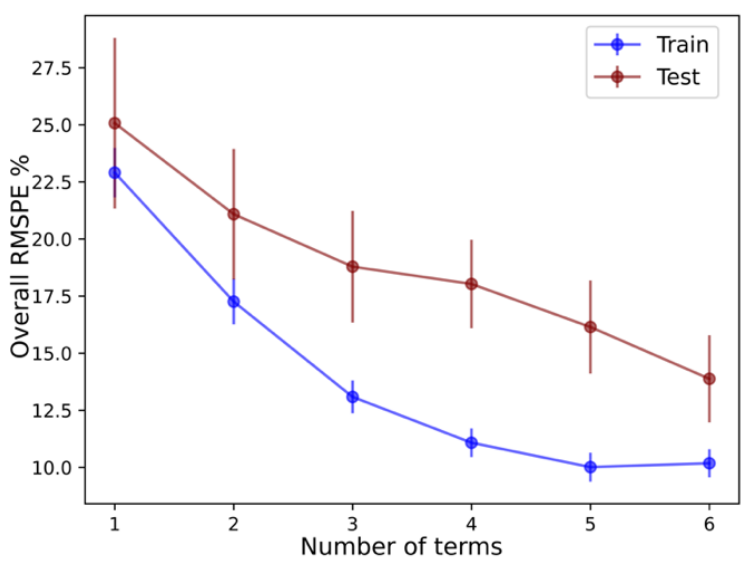

Figure 4 Train and test errors for models with 1 to 6 terms, applied to $\mathrm{C}, \mathrm{O}, \mathrm{CH}_{3}$, and $\mathrm{OH}$, averaged over a hundred test-train splits. The six-term model (Equation 3) is not overfitting on the data. 
We also applied our model to experimental metal-oxide formation energies. As shown in Figure 5 , with an MAE of 0.38 and an $\mathrm{R}^{2}$ of 0.82 , our model does well in predicting and capturing trends in oxide formation energies. The model weights are shown in Table 1. While Figure 5 shows some slight non-linearity, suggesting that slightly higher accuracy is possible using a simple nonlinear model, we use the linear model to preserve high interpretability and easy comparison to the adsorption energy models.

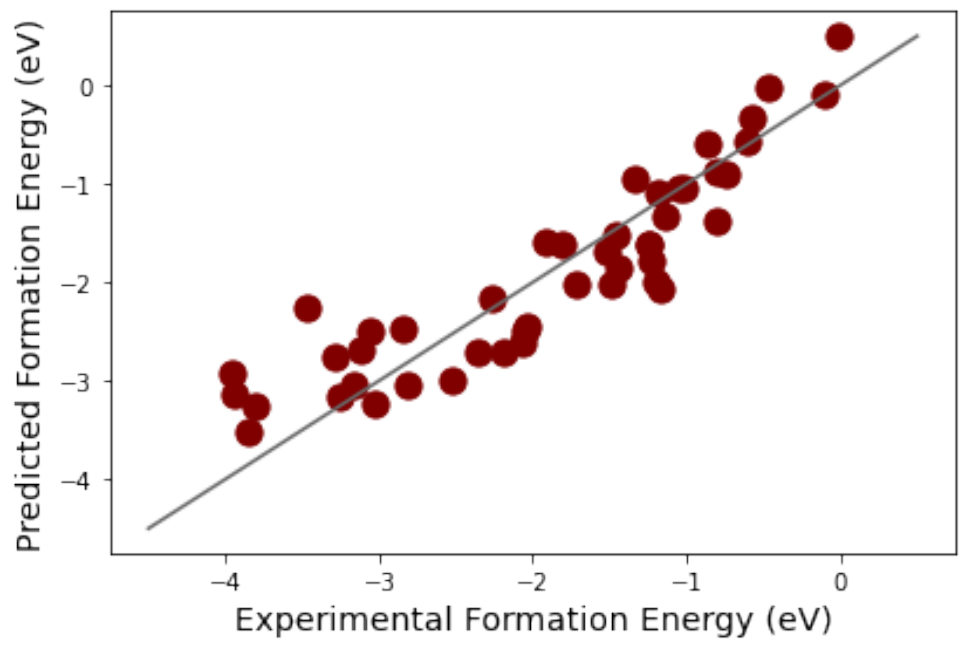

Figure 5 Parity plot between experimental oxide formation energies and model predicted oxide formation energies (Equation 3).

The model weights for $\mathrm{O}, \mathrm{OH}$, and the oxide all have the same sign (see Table 1). This suggests that oxophilicity quantified using adsorption energies and oxophilicity quantified using formation energies are qualitatively similar, as suggested by the correlation between these quantities in Figure 2. We did apply our model to experimental carbide formation energies and found that the model weights had the same signs as $\mathrm{O}$ and $\mathrm{OH}$, as also suggested in Figure 2. However, very few metal carbides have been synthesized experimentally, and this very small dataset makes it difficult to be sure the model is reliable in this case.

Table 1: Model weights for Equation 3 for predicting adsorption energies and formation energies. 


\begin{tabular}{|l|c|c|c|c|c|c|r|}
\hline & $\alpha_{1}$ & $\alpha_{2}$ & $\alpha_{3}$ & $\alpha_{4}$ & $\alpha_{5}$ & $\alpha_{6}$ & $\alpha_{7}$ \\
\hline Model terms & & $\varepsilon_{d}$ & $V_{a s}^{2}$ & $V_{a s}^{2} f$ & $V_{a d}^{2}$ & $V_{a d}^{2} f$ & $R f$ \\
\hline O Adsorption energy & -4.33 & -0.41 & -0.13 & 0.07 & -0.92 & 0.62 & 1.01 \\
\hline OH Adsorption energy & -2.27 & -0.15 & -0.06 & 0.05 & -0.52 & 0.25 & 0.5 \\
\hline $\mathrm{C} \mathrm{Adsorption} \mathrm{energy}$ & -2.86 & -0.55 & -0.17 & 0.12 & -0.18 & -1.94 & 0.72 \\
\hline $\mathrm{CH}_{3}$ Adsorption energy & -0.46 & -0.08 & -0.06 & 0.03 & -0.14 & -0.18 & 0.02 \\
\hline Oxide formation energy & -0.06 & -0.03 & -0.08 & 0.04 & -0.64 & 0.69 & 0.75 \\
\hline metal-dopant selectivity & 58.11 & 2.97 & -1.52 & 0.90 & -18.66 & 32.37 & -12.64 \\
\hline
\end{tabular}

\subsection{Insights from our model.}

Our model gives simple, clear insight into how surface properties affect adsorption. For instance, the higher the $R_{p} f$ (which accounts for the net ionic contributions between the metal and the adsorbate), the weaker the $E_{a d s / \text { form }}$. This makes sense conceptually as metals with higher reduction potentials have a stronger affinity for electrons, hindering charge transfer from the metal to the adsorbate. This is similar to the effect of variations in electronegativity, ${ }^{17,18,28}$ as metals that are highly electronegative tend to have weaker $E_{\text {ads/form }}$. The reduction potential typically increases from left to right across the periodic table (Figure S8), consistent with the generally weaker adsorption on the right side of the periodic table. Based on single-term models and the fitting parameters, $\mathrm{V}_{\mathrm{ad}}{ }^{2}$ is generally the most important term for energetic trends. The behavior of $\varepsilon_{d}$ is very consistent with the d-band model ${ }^{22-24}$ and previous work ${ }^{19,20}$ : A higher $\varepsilon_{d}$ leads to stronger $E_{a d s / f o r m}$, as fewer adsorbate-surface antibonding states are occupied. For example, this explains why $\mathrm{Pt}$ adsorbs $\mathrm{OH}$ more strongly than $\mathrm{Pd}$ and more readily forms an oxide, as $\mathrm{Pt}$ has a higher $\varepsilon_{d}$. Generally, $\varepsilon_{d}$ increases from right to left across the periodic table, which explains the relatively stronger adsorption towards the left side of the periodic table.

While the above terms all have the same sign in all of the models (Table 1), the $\mathrm{V}_{\mathrm{ad}}{ }^{2} f$ term is attractive (negative) for C-containing species but repulsive (positive) for O-containing species. 
We attribute this to the deeper valence states of the $\mathrm{O}$ atom, or equivalently to its lone pair, which will contribute more Pauli repulsion, especially for metals with a more full band. ${ }^{20}$ The $\mathrm{V}_{\mathrm{ad}}{ }^{2}$ term is negative for all models, suggesting that this term captures attractive covalent interactions between metal and adsorbate. Hence, the major distinction between oxophilicity and carbophilicity in the d-block is the dependence on $\mathrm{V}_{\mathrm{ad}}{ }^{2} f$, such that an increase in the $d$ coupling yields relatively less oxophilicity and more carbophilicity. This helps to explain why $\mathrm{Cu}$ is more carbophilic than $\mathrm{Zn}$, but $\mathrm{Zn}$ more oxophilic than $\mathrm{Cu}$. It also explains why $\mathrm{Ag}$ is more oxophilic than $\mathrm{Au}$, but $\mathrm{Au}$ more carbophilic than $\mathrm{Ag}$. In each pair, $\mathrm{Cu}$ and $\mathrm{Au}$ have a larger $\mathrm{V}_{\mathrm{ad}}{ }^{2} f$ which is attractive for carbon-containing species but repulsive for oxygen-containing species.

For metals without any $d$ electrons at all ( $\mathrm{Li}, \mathrm{Be}, \mathrm{Na}, \mathrm{Mg}, \mathrm{Al}, \mathrm{K}, \mathrm{Ca})$, the terms involving $d$ electrons go to 0, and Equation 3 reduces to Equation 4. Therefore, adsorption on these metals is characterized by the metal's ionic properties and the coupling strength between the adsorbate and the metal's $s$ states.

$$
E_{a d s / f o r m}=\alpha_{1}+\alpha_{3} V_{a s}^{2}+\alpha_{4} V_{a s}^{2} f+\alpha_{7} R_{p} f
$$

Equation 4 explains why Li is more oxophilic than Al, but less carbophilic. Li has a significantly lower $\mathrm{R}_{\mathrm{p}} f$ value than $\mathrm{Al}$, leading to higher oxophilicity due to the large value of $\alpha_{7}$ for $\mathrm{O} / \mathrm{OH} /$ oxides. However, in the case of carbophilicity, the value of $\alpha_{7}$ is quite small, making the $\mathrm{V}_{\mathrm{as}}^{2} f$ a more dominant term. A higher $\mathrm{V}_{\mathrm{as}}^{2} f$ leads to weaker bonding. Hence, $\mathrm{Al}$ is more carbophilic than Li because $\mathrm{Al}$ has a lower $\mathrm{V}_{\text {as }}^{2} f$.

Both oxophilicity and carbophilicity decrease down the group for alkali metals but increase down the group for alkaline-earth metals. This trend can be seen in Figure 1, and it can be directly explained by $\mathrm{V}_{\mathrm{as}}{ }^{2}$ (Figure $\mathrm{S} 10$ ). Generally, unlike $\mathrm{V}_{\mathrm{ad}}{ }^{2}, \mathrm{~V}_{\mathrm{as}}{ }^{2}$ increases from left to right within the 
row of each block and decreases down the groups (Figure S4). The value of $V_{\text {as }}^{2} f$ also has different implications depending on how filled the valence orbital of the metal is. For instance, we expect significant repulsion between a filled band and the adsorbate states. Hence, as $V_{\text {as }}^{2} f$ increases, attractive hybridization dominates for the alkali metals, but Pauli repulsion becomes important for the alkaline-earth metals. Additionally, the $\mathrm{R}_{\mathrm{p}} f$ term also captures the adsorption trends of alkaline-earth metals ( $\mathrm{R}_{\mathrm{p}} f$ decreases down the group translating into stronger adsorption) but fails to capture the adsorption trends of the alkali metals.

\subsection{Application}

Previous work ${ }^{2-4}$ has shown that the selectivity of metal catalysts towards ethylene oxide (the desired product) in ethylene epoxidation can be improved by adding metal dopants. To promote selectivity, it has been suggested that the metal dopant should bind strongly to oxygen relative to carbon but should not readily form an oxide. ${ }^{2,19}$ To test this as a design principle, we subtracted the methyl adsorption energy from the hydroxyl adsorption energy, and also examined the oxide formation energy. These factors can indeed explain previously calculated selectivities for various dopants added to $\mathrm{Ag}(100)$ (Figure 6a). In particular, metals with weak oxide formation energies but strong hydroxyl adsorption (relative to methyl) tend to give higher selectivity. Next, we applied this rationale to screen other metal dopants for ethylene epoxidation, as shown in Figure 6b. We observe some potential candidates such as alkali metals, $\mathrm{Tl}, \mathrm{Pb}$, and $\mathrm{Bi}$. Some of these candidates are consistent with literature, ${ }^{50-52}$ as alkali metals have been shown to improve the selectivity of Ag for this reaction. This fast screening shows the utility of having a pre-computed database of different measures of oxophilicity and carbophilicity, as given in this work, for quickly identifying promising candidates for a given application. 
We have thus established the interconnected relationship between the metal dopant's electronic properties, its oxophilic/carbophilic tendencies, and the calculated selectivity for ethylene epoxidation as shown in Figure 6c (blue arrow). However, understanding the direct relationship between the dopant's electronic properties and its selectivity could be very useful (Figure 6c, red arrow). This relationship may allow for further improvements of these doped-metal catalysts based on rational design. To find this relationship, we fit our model (Equation 3) directly to the silver-dopant selectivities as shown in Figure 6d. With an $R^{2}$ value of 0.70 , we see that our model is effective in predicting selectivity. After careful investigation of the model weights (Table 1), we found that $\mathrm{V}_{\mathrm{ad}}{ }^{2} f$ was the dominant term for metals that contain $d$ electrons (either in their core or valence shell) and thus is primarily responsible for trends in the dopant's selectivity. This is consistent with the finding that $\mathrm{Vad}^{2} f$ is the major term that controls oxophilicity relative to carbophilicity. For s block metals, $\mathrm{V}_{\text {as }}^{2} f$ controls trends in selectivity. Hence, transition metals with high $\mathrm{V}_{\text {ad }}{ }^{2} f$ and s block metals with low $\mathrm{V}_{\text {as }}^{2} f$ are good dopant candidates for this reaction. 

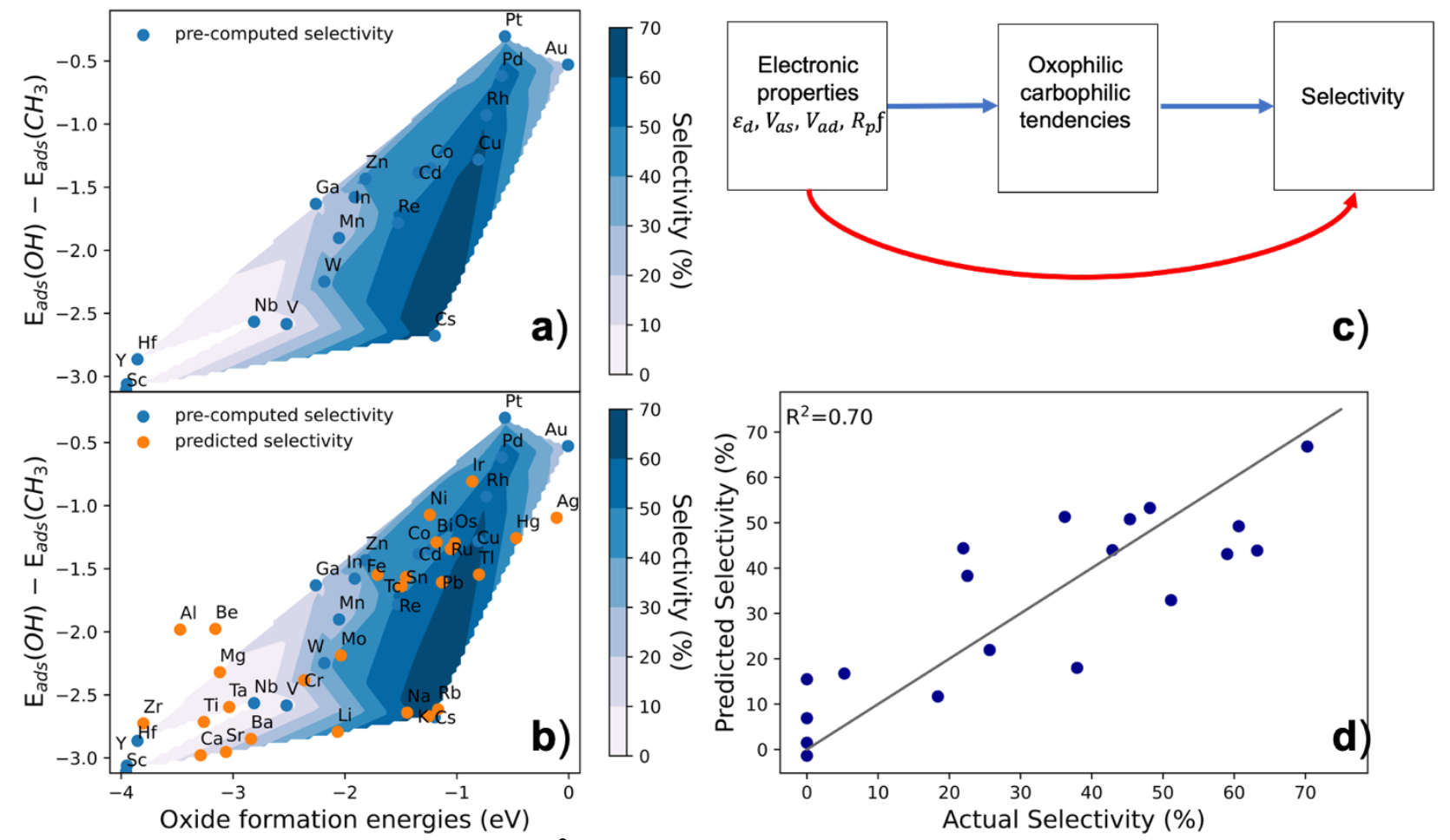

c)

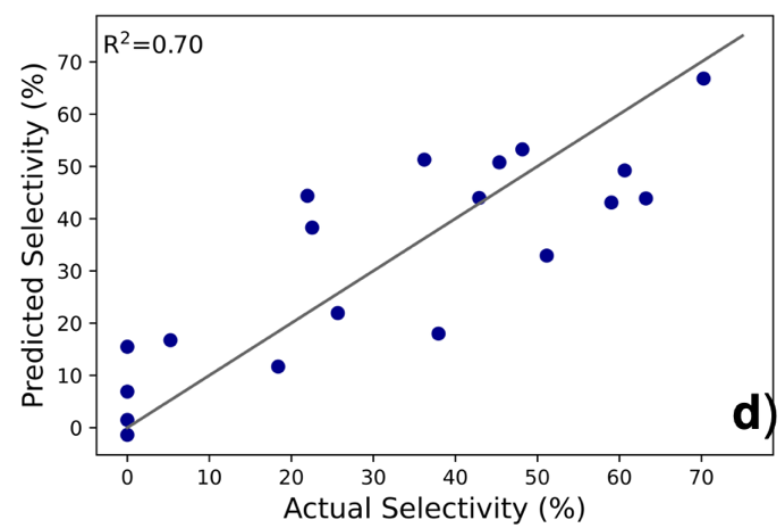

Figure 6. (a) Calculated selectivities ${ }^{2}$ for ethylene epoxidation on $\operatorname{Ag}(100)$ doped with various metals as a function of the difference in methyl and hydroxyl binding strengths and oxide formation energies. The metals for which we have data are shown, with selectivities interpolated and shown as a contour plot. Dopants that are more oxophilic than carbophilic but do not readily form oxides have higher selectivities. (b) Screening additional metal dopants using the contour plot from Figure 6a. Some promising candidates were identified, such as alkali metals, Tl, Pb, and Bi. (c) The interconnected relationship between the metal dopant's electronic properties, its oxophilic/carbophilic tendencies, and its selectivity for ethylene epoxidation has been established (blue arrow). To improve catalytic design, it might be useful to establish the direct relationship between the dopant's electronic properties and its selectivity (red arrow). (d) Parity plot between the actual selectivities and the model-predicted selectivities.

\section{Conclusion}

We developed a general, interpretable model that accurately explains and predicts oxophilicity and carbophilicity of transition metals and main group metals all together. The model is accurate for methyl, carbon, oxygen, and hydroxyl adsorption energies, as well as oxide formation energies, and explains periodic trends for these quantities. The model has an ionic term $\left(\mathrm{R}_{\mathrm{p}} \mathrm{f}\right)$, as well as attractive covalent terms $\left(\mathrm{V}_{\mathrm{as}}^{2}, \mathrm{~V}_{\mathrm{ad}}{ }^{2}\right)$, a repulsive term $\left(\mathrm{V}_{\mathrm{as}}{ }^{2} \mathrm{f}\right)$, and a term that is 
attractive for species without a lone pair and repulsive for species with a lone pair $\left(\mathrm{V}_{\mathrm{ad}}{ }^{2} \mathrm{f}\right)$. Hence, in all cases, a lower $R_{\mathrm{p}} f$, higher $\mathrm{V}_{\text {as }}^{2}$, lower $\mathrm{V}_{\text {as }}^{2} \mathrm{f}$, and higher $\mathrm{V}_{\text {ad }}^{2}$ lead to stronger bonding, while a high $\mathrm{V}_{\mathrm{ad}}{ }^{2} f$ leads to higher carbophilicity but weaker oxophilicity. The model has good accuracy and explains trends across the periodic table. We showed the utility of our model by first establishing the inter-connected relationship between a metal dopant's electronic properties, its oxophilic/carbophilic tendencies, and its selectivity for ethylene epoxidation. Next, with our model, we established a direct relationship between the metal dopant's electronic properties and its selectivity for ethylene epoxidation. We found that transition metals with high $\mathrm{V}_{\mathrm{ad}}{ }^{2} f$ and $\mathrm{s}$ block metals with low $\mathrm{V}_{\text {as }}^{2} f$ are good dopant candidates for this reaction.

\section{Conflicts of Interest}

The authors declare no conflicts of interest.

\section{Acknowledgements}

We acknowledge the support from the U.S. - Israel Center for Fossil Fuels, administered by the BIRD foundation, as well as support from Tulane University. Computational resources for this work were provided by Tulane University's Technological services and the Louisiana Optical Network Infrastructure (http://www.loni.org).

\section{References}

1 F. Studt, F. Abild-Pedersen, T. Bligaard, R. Z. Sørensen, C. H. Christensen and J. K. Nørskov, Science, 2008, 320, 1320-1322.

2 H. Xu, L. Zhu, Y. Nan, Y. Xie and D. Cheng, ACS Catal., 2021, 11, 3371-3383. 
3 M. O. Özbek and R. A. Van Santen, Catal. Letters, 2013, 143, 131-141.

4 S. Chavadej and T. Suttikul, Plasma Chem. Plasma Process., 2021, 41, 265-288.

5 M. Aresta, Coord. Chem. Rev., 2017, 334, 150-183.

6 X. Lan and T. Wang, ACS Catal., 2020, 10, 2764-2790.

7 M. Luneau, J. S. Lim, D. A. Patel, E. C. H. Sykes, C. M. Friend and P. Sautet, Chem. Rev., 2020, 120, 12834-12872.

8 G. Jones, J. G. Jakobsen, S. S. Shim, J. Kleis, M. P. Andersson, J. Rossmeis1, F. AbildPedersen, T. Bligaard, S. Helveg, B. Hinnemann, J. R. Rostrup-Nielsen, I. Chorkendorff, J. Sehested and J. K. Nørskov, J. Catal., 2008, 259, 147-160.

9 M. M. Montemore, E. D. Cubuk, J. E. Klobas, M. Schmid, R. J. Madix, C. M. Friend and E. Kaxiras, Phys. Chem. Chem. Phys., 2016, 18, 26844-26853.

10 B. Hammer and J. K. Nørskov, Nature, 1995, 376, 238-240.

11 M. D. Argyle and C. H. Bartholomew, Catalysts, 2015, 5, 145-269.

12 S. Shan, J. Li, Y. Maswadeh, C. O’Brien, H. Kareem, D. T. Tran, I. C. Lee, Z. P. Wu, S. Wang, S. Yan, H. Cronk, D. Mott, L. Yang, J. Luo, V. Petkov and C. J. Zhong, Nat. Commun., 2020, 11, 1-9.

13 M. M. Montemore, M. A. Van Spronsen, R. J. Madix and C. M. Friend, Chem. Rev., 2018, $118,2816-2862$.

14 A. Kolker, Econ. Geol., 1982, 77, 1146-1158.

15 Clifford Frondel, Am. Mineral., 1943, 28, 521-535.

16 C. Ma and A. E. Rubin, Am. Mineral., 2019, 104, 1351-1355.

17 K. A. Moltved and K. P. Kepp, J. Phys. Chem. C, 2019, 123, 16.

18 K. P. Kepp, Inorg. Chem, 2016, 55, 11. 
19 M. M. Montemore and J. W. Medlin, J. Phys. Chem. C, 2014, 118, 2666-2672.

20 M. M. Montemore and J. W. Medlin, J. Am. Chem. Soc., 2014, 136, 9272-9275.

21 R. García-Muelas and N. López, Nat. Commun., 2019, 10, 1-7.

22 B. Hammer, Y. Morikawa and J. K. Nørskov, Phys. Rev. Lett., 1996, 76, 2141-2144.

23 B. Hammer, Top. Catal., 2006, 37, 3.

24 B. Hammer and J. K. Nørskov, in Advances in Catalysis, eds. B. C. Gates and H. Knozinger, Academic Press, 2000, vol. 45, pp. 71-129.

25 M. Andersen, S. V Levchenko, M. Scheffler and K. Reuter, ACS Catal., 2019, 9, 27522759.

26 G.-C. Wang, J. Li, X.-F. Xu, R.-F. Li and J. Nakamura, J Comput Chem, 2005, 26, 871878.

27 A. M. Deml, R. O’Hayre, C. Wolverton and V. Stevanović, Phys. Rev. B, 2016, 93, 085142.

28 A. M. Deml, A. M. Holder, R. P. O’Hayre, C. B. Musgrave and V. Stevanović, J. Phys. Chem. Lett., 2015, 6, 1948-1953.

29 V. Stevanović, S. Lany, X. Zhang and A. Zunger, Phys. Rev. B - Condens. Matter Mater. Phys., 2012, 85, 115104.

30 C. F. Dickens, J. H. Montoya, A. R. Kulkarni, M. Bajdich and J. K. Nørskov, Surf. Sci., $2019,681,122-129$.

31 Y. Mao, H. Yang, Y. Sheng, J. Wang, R. Ouyang, C. Ye, J. Yang and W. Zhang, ACS Omega, 2021, 6, 14533-14541.

32 D. You, S. Ganorkar, S. Kim, K. Kang, W. Y. Shin and D. Lee, Scr. Mater., 2020, 183, 15. 
33 C. J. Bartel, A. Trewartha, Q. Wang, A. Dunn, A. Jain and G. Ceder, npj Comput. Mater., 2020, 6, 1-11.

34 M. M. Montemore and J. W. Medlin, J. Phys. Chem. C, 2013, 117, 2835-2843.

35 M. M. Montemore, C. F. Nwaokorie and G. O. Kayode, Catal. Sci. Technol., 2020, 10, $4467-4476$.

36 S. Kirklin, J. E. Saal, B. Meredig, A. Thompson, J. W. Doak, M. Aykol, S. Rühl and C. Wolverton, npj Comput. Mater., 2015, 1, 1-15.

37 J. A. C. Broekaert, Anal. Bioanal. Chem., 2015, 407, 8943-8944.

38 Walter A. Harrison, Electronic Structure and the Properties of Solids: The Physics of the Chemical Bond, Dover Publications, New York, 1989.

39 G. Kresse and J. Hafner, Phys. Rev. B, 1993, 47, 558.

40 G. Kresse and D. Joubert, Phys. Rev. B, 1999, 59, 11-19.

41 J. P. Perdew, J. A. Chevary, S. H. Vosko, K. A. Jackson, M. R. Pederson, D. J. Singh and C. Fiolhais, Phys. Rev. B, 1992, 46, 6671-6687.

42 P.E.Blöchl, Phys. Rev. B, 1994, 50, 17953-17979.

43 Hendrik J. Monkhorst, Phys. Rev. B, 1976, 13, 5188-5192.

44 F. Abild-Pedersen, J. Greeley, F. Studt, J. Rossmeisl, T. R. Munter, P. G. Moses, E. Skúlason, T. Bligaard and J. K. Nørskov, Phys. Rev. Lett., 2007, 99, 4-7.

45 D. Y. Chung, H. Il Kim, Y. H. Chung, M. J. Lee, S. J. Yoo, A. D. Bokare, W. Choi and Y. E. Sung, Sci. Rep., 2014, 4, 7450.

46 M. M. Montemore and J. W. Medlin, Catal. Sci. Technol., 2014, 4, 3748-3761.

47 A. Nilsson, L. G. M. Pettersson, B. Hammer, T. Bligaard, C. H. Christensen and J. K. Nørskov, Catal. Letters, 2005, 100, 111-114. 
48 A. P. Monasterial, C. A. Hinderks, S. Viriyavaree and M. M. Montemore, J. Chem. Phys., 2020, 153, 111102.

49 S. Nayak, S. Bhattacharjee, J.-H. Choi and S. C. Lee, J. Phys. Chem. A, 2019, 124, $247-$ 254.

50 R. B. Grant and R. M. Lambert, Langmuir, 1985, 1, 29-33.

51 M. A. Salaev, Mol. Catal., 2021, 507, 111574.

52 M. Huš and A. Hellman, J. Catal., 2018, 363, 18-25. 